\title{
REPENSANDO LOS CONCEPTOS DE SUBSUNCIÓN, PLUSVALOR Y TRABAJO DE AUDIENCIA EN EL CAPITALISMO DIGITAL
}

\section{RETHINKING THE CONCEPTS OF SUBSUMPTION, SURPLUS VALUE AND AUDIENCE LABOUR IN DIGITAL CAPITALISM}

\section{Eduardo Molina Campano}

Laboratorio de Ideas y Prácticas Políticas

Universidad Pablo de Olavide (Sevilla)

edula7@hotmail.com

Recibido: noviembre de 2018

Aceptado: diciembre de 2018

Palabras clave: Subsunción, plusvalor, redes sociales, renta, trabajo de audiencia, prosumidor.

Keywords: Subsumption, surplus-value, social media, rent, audience labour, prosumer.

Resumen: En este artículo se pretende analizar el concepto de subsunción en relación con la evolución del trabajo y el plusvalor centrándonos en el llamado trabajo de audiencia como aquella actividad constante de los usuarios de los medios de comunicación digital y de las redes sociales del capitalismo contemporáneo. Para algunos autores esta actividad produce directamente plusvalor en abstracto y debe ser considerado como un trabajo productivo. En las conclusiones intentamos aportar algunas ideas a este debate sobre la cuestión.

Abstract: This article aims to analyze the concept of subsumption in relation to the evolution of labour and surplus value focusing on the so-called audience labour as that constant activity of the users of the digital media and social networks of contemporary capitalism. For some authors this activity directly produces surplus value in abstract and should be considered as productive labour. In the conclusions we try to contribute some ideas to this debate on the issue.

\section{Introducción}

Uno de los tantos efectos de la crisis económica del 2008 ha sido el reimpulso, en el sector académico, del análisis neomarxista en la economía política ${ }^{1}$ en general. De ma-

1. En el período comprendido desde el 2008 hasta el 2014, los artículos sobre Marx o el marxismo en revistas indexadas de Ciencias Sociales, casi se triplicaron con respecto a la década anterior. (Fuchs \&Fisher, 2015, 6) 
nera que ciertos grupos de investigadores y profesores en el ámbito internacional comenzaron a analizar críticamente, pero vinculados a la perspectiva de la ley del valor-trabajo de Marx, los cambios más recientes en el mundo del trabajo. Ya antes la escuela autonomista italiana había emprendido estos estudios. Esta escuela permanece más viva que nunca y se encuentra inmersa en franco y sano debate con estos nuevos grupos de investigación.

Estos análisis han tenido como eje vertebral al espacio comunicacional de internet que ha fungido como una de las bases principales del actual crecimiento de las fuerzas productivas en términos globales. (Luego de la crisis sectorial de las punto.com y la subsiguiente crisis financiera global).

Este crecimiento relacionado con el espacio de la red se centra en la industria informacional, tanto física como inmaterial, es decir: hardware, software, semiconductores, antenas para la propia infraestructura de internet, publicidad, editoriales y radiodifusión. Esta industria informacional se ha convertido en uno de los principales terrenos de inversión por parte del capital transnacional junto al sector aún mayoritario de las FIRE, (finanzas, seguros y bienes raíces), compitiendo casi en igualdad de activos con el sector de la movilidad (infraestructura de transporte, petróleo, gas y vehículos)².

Estos nuevos investigadores, junto a los autonomistas italianos, se enfrentan a una realidad del trabajo que difiere significativamente de la época industrial en sus

2. Para revisar estos datos porcentuales recomendamos el artículo de Christian Fuchs (2016) Digital Labour and Imperialism: 4 y 5. Disponible en: monthlyreview.org/2016/01/01/digital-laborand-imperialism/ diferentes fases, asumiendo así el interesante reto de actualizar la teoría del plusvalor de Marx. El objeto de este artículo es precisamente publicitar, al menos parcialmente, este debate que se está desarrollando y realizar un análisis del mismo.

\section{La evolución del concepto de subsunción}

\subsection{Introducción}

Con la crisis económica de 1973 entra en crisis la fase fordista del capitalismo monopólico industrial auspiciado tras la Segunda Guerra Mundial. Esta etapa histórica estuvo definida por la intervención del Estado en la economía de los estadosnación dominantes con el objeto de regular la demanda efectiva de los consumidores. Esto significaba en la práctica un alto grado de empleo fabril con buenos salarios para garantizar la realización de la plusvalía contenida en las mercancías y lanzadas al mercado, a través de las empresas de comercialización, por los grandes monopolios de la producción.

A este período post crisis se le empezó a denominar en el argot económico como postfordismo aunque no quedaba muy claro, al tratarse de una transición, cuál sería el nuevo paradigma productivo. Esta transición es lo que algunos autores Ilamaron fase de descenso de la onda larga económica (Mandel, 1983) o la última transición hacia el Imperio (Negri \& Hardt, 2002). Sin embargo, no será hasta el final de la primera Guerra del Golfo (febrero de 1991), en el contexto de la desintegración de la Unión Soviética (de marzo de 1990 hasta diciembre de 1991), cuando comience a delinearse este nuevo patrón productivo que va más allá del sistema 
toyotista de producción ${ }^{3}$ implementado y que aún sigue vigente.

Este paradigma se iría identificando poco a poco con la industria del conocimiento tras un período previo de innovaciones en los campos del transporte, el lenguaje y la comunicación. Innovaciones que llevaron al desarrollo de internet como fuente de difusión y producción de la información, contenidos y servicios. Esta revolución basada en la velocidad de la comunicación ha sido la base para el aumento de la productividad actual del capitalismo contemporáneo al provocar otra vuelta de tuerca en el achicamiento del tiempo de trabajo socialmente necesario (valor) para la producción de las mercancías.

Se trata de una forma de subordinación más amplia que en la era industrial pues se extiende a toda la sociedad de consumo y del conocimiento. Un subsunción que explota al individuo indirectamente hasta en su tiempo libre desdibujando las fronteras fordistas del tiempo de trabajo y el tiempo de ocio. La vida se mercantiliza convirtiendo cualquier valor de uso en valor de cambio.

\subsection{La subsunción formal}

Es sabido que el proceso de trabajo como creador de valores de uso destinados a

3. Sistema de producción en cadena inventada por el ingeniero japonés Taiichi Ohno. Se basa en el precepto "justo a tiempo" que busca producir solo aquello que se necesita y en el tiempo preciso, reduciendo la oferta de bienes a la inversa de lo que ocurría en el período fordista. Esto trajo como consecuencia la elevación de los precios de las mercancías y el despido de un gran porcentaje de mano de obra que pasa a la temporalidad estructural al servicio de las fluctuaciones del mercado. Este sistema aún sigue vigente y está integrado en el nuevo paradigma del capitalismo cognitivo. satisfacer necesidades directas de la población ha sido común a todos los modos de producción. En los comienzos del capitalismo este proceso de trabajo empezó a mistificarse (fetichizarse) a través de lo que Marx denominó la subsunción del trabajo en el capital, un concepto de origen latino y de uso inglés y alemán (subsumption-subsumtion) que significa subordinación e inclusión al mismo tiempo.

De acuerdo con Marx (1982, 2010, 2013) explicitado en los Manuscritos Económicos de 1861-63, y en los capítulos VI (inédito) y XIV del libro I del Capital, el período de la subsunción formal del trabajo está comprendido entre los inicios del capitalismo mercantil a fines del siglo XVI hasta finales del siglo XVIII. Dos siglos de transición donde "el proceso de trabajo se convierte ahora en instrumento del proceso de valorización" ${ }^{4}$, esto es, de la creación de plusvalía. El taller artesanal libre y urbano resultó ser el centro de desarrollo de este fenómeno. Todo el plusvalor como expresión del plustrabajo impago lo generaba el oficial del taller que empezó a fungir como obrero asalariado manteniendo, sin embargo, el conocimiento y las destrezas previas así como un cierto control sobre las herramientas pero bajo la dirección del pequeño capitalista.

El proceso laboral, desde el punto de vista tecnológico, se efectuaba exactamente como antes sólo que ahora subordinado al capitalista y no al maestro corporativo. Los capitalistas, aún pequeños, eran los mismos maestros que a veces les tocaba también trabajar para suplir todas las ne-

4. Marx (2013) El Capital, Capítulo VI (inédito):15. Este capítulo fue excluido por Marx en la publicación del tomo I del Capital en 1867 y no fue publicado hasta 1933 en la Unión Soviética. El nombre original es "Resultados del proceso inmediato de producción". 
cesidades. La diferencia entre el trabajo formalmente subsumido en el capital y el modo de producción anterior se manifestaba en el volumen de capital invertido por el capitalista individual.

El reemplazo de la esclavitud, la servidumbre, el vasallaje y las formas patriarcales por la nueva subordinación del oficialobrero al capital tan solo modificaba la forma de la explotación más no el contenido. Esta se volvió más libre, formalmente voluntaria, puramente económica (Marx, 1983, 2010, 2013). Mientras el esclavo, por ejemplo, recibía un salario mínimo independientemente de su trabajo, el nuevo obrero no lo tenía garantizado y estaba obligado a trabajar y a competir con sus propios compañeros para ser contratado a cambio de la cesión de toda su fuerza y experticia de trabajo si no quería morir de hambre tanto él como su familia.

Algo parecido ocurrió, con sus diferencias, con la liberación del siervo campesino y el oficial corporativo al convertirse en jornaleros y obreros libres asalariados. En todos los casos se perdía la certidumbre de tener asegurada la satisfacción de sus necesidades básicas, a pesar del sometimiento social que sufrían, a cambio de la libertad de movimiento y contratación que les permitía elegir el lugar donde trabajar.

\subsection{La subsunción real}

Con la transición de la subsunción formal a la real, que tuvo como hito rupturista a la Primera Revolución Industrial, el proceso de explotación y extracción de plusvalor pasó de la extensión de la jornada laboral a la intensificación del proceso de trabajo. A este plusvalor Marx lo llama relativo para diferenciarlo del absoluto. Esta revolución fue una consecuencia de la aplicación de la ciencia y la tecnología a la producción inmediata provocando la incorporación de la máquina y aumento exponencialmente las fuerzas productivas. Simultáneamente esta producción a gran escala tendía a conquistar todas las ramas industriales de las que no se había apoderado y en la que aún existía la subsunción formal a pequeña escala o incluso el trabajo artesano independiente. (Marx, 2010, 2013)

En la subsunción real el trabajador individual, que en el taller elaboraba completamente un producto, se convierte ahora en un mero eslabón de un colectivo que trabaja en cooperación a través de la división del trabajo para generar una mercancía que contiene la plusvalía como expresión de todo el plustrabajo impago. Esta transición desde la subsunción formal a la real pasará por tres fases, a saber: desde el sistema de la cooperación simple del taller artesanal precapitalista se pasa a finales del siglo XVIII a la manufactura de la época de Adam Smith donde los trabajadores aún usaban sus propias herramientas, por lo que la subsunción seguía siendo semi formal. Esta manufactura, a mitad del siglo XIX, se transforma en la gran fábrica y el trabajador pierde toda su autonomía en el proceso de trabajo convirtiéndose en un mero apéndice y siervo de la máquina, adaptándose a su ritmo y actuando sin pensar. (Marx, 1982, 2007, 2010, 2013)

Es ahora cuando la forma de extracción del plusvalor pasa a estar definido por la intensidad del ritmo que le impone la máquina al obrero. Ritmo que tiene por objeto acortar el tiempo de trabajo socialmente necesario para la producción de mercancías y la misma reproducción de la fuerza de trabajo, incrementando así la tasa del plusvalor (relativo) al aumentar el tiempo de trabajo impago que es el objetivo final de todo capitalista. El tiempo 
y el cronómetro se convierten en centrales como sistema de medición del trabajo que se transforma en una actividad más abstracta, es decir, desprovista de cualquier elemento de creatividad.

El conocimiento del trabajador es usurpado, externalizado y personificado en la máquina. La relación entre el capital constante, fijo o trabajo muerto (la máquina) y el capital variable o trabajo vivo (el obrero), cambia con relación a la subsunción formal porque el trabajador es desprofesionalizado, expropiándole el conocimiento y la habilidad del antiguo artesano que queda personificada y coagulada en la máquina.

Existe por tanto un traslado del conocimiento desde el trabajo vivo al muerto. E capital ahora parece tender a su autovalorización, aumentando la mistificación del valor al dejar de depender de las destrezas de los antiguos oficiales. Durante el siglo XX esta subsunción real basada en la producción material llega a su apogeo con el sistema Taylorista y Fordista, que separaron completamente las tareas de concepción de las de ejecución. El primero llevó a la crisis del 1929 y el segundo al New Deal de la segunda postguerra como estrategia de estabilización del sistema (a través del fomento de la demanda efectiva desde los Estados) y al mismo tiempo atenuar la lucha de clases que la revolución bolchevique había incentivado en todo el mundo industrializado.

\subsection{La subsunción total actual}

Los neomarxistas autonomistas italianos como Negri, Virno, Lazzarato, Marazzi entre otros, desde los años 70, 80 y 90 comenzaron a desarrollar el concepto de Marx (2007) de Intelecto General como la nueva fuerza de trabajo productiva ba- sada en el conocimiento social haciendo hincapié en una diferencia, a saber: mientras Marx lo relacionaba con el capital fijo, es decir, con el conocimiento científico técnico coagulado en las máquinas, los autonomistas lo relacionan con el trabajo vivo, es decir, con los trabajadores del conocimiento que producen mercancías inmateriales relacionadas con los aspectos ligüísticos - relacionales - comunicativos y que son independientes del capital propiamente dicho. De ahí su relativa autonomía con respecto al capital, hecho que no ocurría durante la subsunción real fordista. (Negri \& Lazzarato, 2001).

Esta transición post subsunción real, que llega hasta nuestros días, ha sido definida por algunos autores como subsunción del intelecto general (Vercellone, 2007) para hacer énfasis en la subordinación del conocimiento al capital; subsunción de la vida (Fumagalli, 2015) para darle una connotación más holística al sometimiento del trabajo y no reducirlo exclusivamente al conocimiento; o subsunción de la sociedad al capital (Negri, 2001, 2002, 2004, 2011) para ensanchar al sujeto (el obrero social, la multitud) en dicha inclusión y subordinación.

Quizás un término más lógico con la secuencia propuesta por Marx (2010), afirmamos, sea el de subsunción total, un concepto que indicaría que la subsunción se ha ampliado al ámbito de la vida personal borrando las fronteras entre lo laboral y lo recreativo. Además es una subordinación que implica la combinación de la subsunción formal con la real. Formal porque el conocimiento es una destreza autónoma al capital, que reside en el cerebro humano y que es luego (el conocimiento) mercantilizado e integrado de una manera similar a la que se llevó a cabo con los oficiales artesanos en los inicios del capitalismo. De 
ahí la potencialidad de la autonomía del trabajo actual con respecto al capital que defienden los autonomistas.

Este desarrollo del Intelecto General como conocimiento social adquirido, Vercellone (2007) lo explica como una consecuencia de las políticas del Estado de Bienestar (Welfare State) en materia educativa y de salario indirecto, pues permitió conceder el tiempo libre suficiente a una parte de la población para formarse en una gran cantidad de destrezas lingüísticas y relacionales transformándolos en "productivos" en sí mismos. Es decir, el capital variable como trabajo vivo se convierte al mismo tiempo en capital fijo (el cerebro) independiente pero al mismo tiempo vinculado y casi fusionado a las nuevas tecnologías de la comunicación.

Esta nueva subsunción formal que se apropia de estos valores de uso (las habilidades cognitivas y relacionales) para convertirlos en valores de cambio, viene acompañada de la subsunción real de forma combinada pues el trabajo material industrial no desaparece sino que se reduce y se transforma en los países avanzados (sistema toyotista) para trasladar el sistema fordista a otras regiones más rentables para el capital.

Esta subsunción total de la vida vinculada a las nuevas tecnologías dinámicas requiere de una gobernanza específica para controlar a la ciudadanía. Fumagalli (2015), señala que estos instrumentos son la deuda individual y la precariedad en el empleo. Estos serían las dos principales herramientas disciplinarias de la actual sociedad de control para regular la psicología de los individuos de una forma más efectiva que el mando jerárquico existente en la antigua fábrica. Un control indirecto que genera auto represión, competitividad al máximo nivel y sentimiento de culpa. (Fumagalli, 2015). Añadiríamos el control de la información personal a través de las redes sociales y de los buscadores como Google que fungen como el gran hermano orweliano que lo ve y lo registra todo.

Ahora bien, esta subsunción total que no solo subordina al trabajo sino a toda la sociedad como dice Negri (2001, 2002, 2004, 2011) no tiene por qué implicar la desaparición de la ley del valor-trabajo como apuntan en general los autonomistas 5 . En este sentido y estando de acuerdo con Jakob Rigi (2015), si bien es cierto que la ley del valor es socavada parcialmente en los países avanzados tanto por la dificultad de cuantificar el plusvalor generado por la sociedad implicada en las actividades de producción de conocimiento como por el achicamiento del tiempo de trabajo socialmente necesario, la ley del valor en realidad se amplía globalmente al extenderse a otras regiones del planeta como China donde la subsunción real fordista sigue en pie. Si se pierde la perspectiva mundial desde el punto de vista del funcionamiento de la cadena de valor es difícil comprender el rol interdependiente de unas economías frente a otras.

\section{El trabajo de audiencia en los medios de comunicación digitales}

\section{I lntroducción}

Luego de reseñar y analizar brevemente la historia de la subsunción del trabajo en el capital estamos en mejores condiciones

5. Ver las propuestas de Fumagalli (2015) o Negri (s.f) en torno a la teoría del valor-vida o el valor-afecto respectivamente. 
de reflexionar sobre el plusvalor en el sector digital del capitalismo contemporáneo. Si se toman como válidas, sin entrar en matices diferenciales, las afirmaciones de la escuela autonomista italiana, la subsunción actual estaría definida por una combinación de subsunción formal y real teniendo como base hegemónica de explotación no al trabajo físico fabril (que se traslada a la periferia) sino al conocimiento humano que funge como fuerza productiva directa (intelecto general) fusionada con las nuevas tecnologías.

La pregunta es cómo esta nueva relación social (intelecto general/capital) produce en algunos casos plusvalor, renta o ganancias especulativas para las corporaciones de la industria de la información y la comunicación digital.

\subsection{El concepto del Trabajo de Audiencia}

El concepto clave que algunos investigadores ponen sobre la mesa para entender este fenómeno de la subsunción total que se expresa más claramente en los países dominantes gira en torno a la noción del "trabajo de audiencia". Esta idea fue introducida en un primer momento por Dallas Smythe (1977) en su famoso artículo Communications: Blindspot of Western Marxism (Comunicaciones: el agujero negro del marxismo occidental). En este escrito Smythe realiza una crítica a la visión culturalista del marxismo occidental ${ }^{6}$ con relación a la industria de la información y

6. Conjuntos de autores diversos que se distinguen del marxismo soviético y que en general se centraron en cuestiones superestructurales relacionadas con la reproducción ideológica dejando de lado las cuestiones económicas en una suerte de rechazo al economicismo ortodoxo soviético. comunicación al tratarla exclusivamente como parte de la superestructura ideológica.

Para ello Smythe (1977) se centra en la cuestión de la publicidad como el instrumento fundamental de los grandes medios para inducir a la "audiencia" a consumir los productos que se anuncian para completar y asegurar la realización del ciclo de la producción, convirtiendo con ello a los usuarios mismos en una mercancía que se vende a las compañías publicitarias. Una mercancía cuyo valor de uso radica en la capacidad para "poner atención" a los anuncios publicitarios. Esta capacidad es la que compran las compañías publicitarias a los dueños de los medios de comunicación masivos con el cebo de la oferta de programas televisivos.

Sin embargo, Graham Murdock (1978) ${ }^{7}$ responderá a Smythe para afirmar que a pesar de los aciertos de la crítica del investigador canadiense "la preocupación de Smythe sobre las relaciones entre comunicación y publicidad le lleva a minusvalorar la función independiente del contenido de los medios a la hora de reproducir las ideologías dominantes." (p: 5) Este déficit Murdock lo llamará irónicamente "Ios agujeros negros de Smythe" para utilizar la misma metáfora usada por este en el título de su artículo.

Esta polémica que incentivó el desarrollo de una economía política de la comunicación se ha reanimado en los años recientes a colación de la industria digital en internet y en las redes sociales como los nuevos medios de comunicación e información de masas. Para indagar sobre esta discusión nos centraremos en tres

7. Blidspots About Western Marxim: A Reply to Dallas Smythe. 
artículos recientes escritos por: Brice Nixon, Christian Fuchs, y Eran Fisher ${ }^{8}$.

\subsection{El debate}

Brice Nixon (2015) afirma que el trabajo de audiencia debe ser reconceptualizado para superar teoréticamente el listón que había dejado Smythe. Para Nixon la relación fundamental en la economía política de la comunicación no es entre los anunciantes y la audiencia sino entre los dueños de los medios y la audiencia. Nixon hace un intento de analogía entre el proceso de trabajo descrito por Marx (18672010) en el primer volumen del capital con el proceso de trabajo relacionado con la industria cultural en general. Compara el papel que tienen los dueños de los medios de comunicación con el rol del propietario de la tierra para establecer algunos puntos en común y algunas diferencias. Señala que el propietario de una industria cultural concede el derecho de uso del contenido cultural que posee a cambio de un pago en forma de renta. Una renta que o bien proviene directamente del salario de los trabajadores o bien de una parte del plusvalor apropiado por otro capitalista como por ejemplo los anunciantes. Los anunciantes pagan un impuesto al dueño del medio de comunicación (para promocionar sus productos a la audiencia), que es lo que permite a la audiencia poder acceder libremente al contenido cultural.

8. Brice Nixon es profesor del Departamento de Comunicación de la Universidad de La Verne en EEUU. Christian Fuchs es director del Instituto de Investigación de Medios de Comunicación y profesor de la Universidad de Westminster, Reino Unido y Eran Fischer es profesor del Departamento de Sociología, Ciencias Políticas y Comunicación de la Universidad Abierta de Israel.
Nixon afirma que esto último es la forma más común de explotar a la audiencia que funge como un trabajador productivo para el capital porque sus actividades de consumo cultural generan un valor para el capitalista de los medios ya sea por la venta directa de una mercancía cultural o por el impuesto que recibe del anunciante. Sin embargo, se podría inferir que el capitalista de la comunicación se comporta como un terrateniente al explotar el trabajo de la audiencia (su capacidad de percepción y decodificación) sin pagar un salario al usuario por el tiempo de "trabajo" que invierte en el consumo cultural y que acrecienta el capital de los medios. Es como un trabajo abstracto que se realiza de forma más o menos pasiva (percepción y decodificación) a través del uso de los instrumentos de "trabajo" naturales que posee el individuo como son la vista y el oído. Estos medios naturales junto a los artificiales definidos por los dispositivos tecnológicos actuales, fungirían como los instrumentos de trabajo del capital digital cuyo objeto no es otro que la producción de cultura en un sentido amplio del término.

Ahora bien, la producción y las características de este plusvalor generado es difícil de captar, medir y definir pues está completamente mistificado. Nixon (2015) afirma que "la extracción del interés de los anunciantes es un proceso de explotación indirecta del trabajo de audiencia por el capital comunicativo porque el plusvalor es tomado del anunciante y no del trabajador audiencia." (p:111) (Traducción propia)

Estamos de acuerdo con ese análisis aunque Nixon no dice de dónde sale realmente ese plusvalor que es transferido desde los anunciantes al dueño del medio de comunicación. Al no responder esa pregunta se dificulta entender el funcionamiento del trabajo de audiencia como 
trabajo productivo en sí mismo, es decir, como generador directo de plusvalor en un sentido estricto del término.

Jhally and Livant ${ }^{9}(1986)$ trataron de hacerlo argumentando que ver televisión es una forma de trabajo que genera plusvalor cuando la audiencia ve más anuncios de los necesarios para cubrir los gastos de los programas que se emiten en el medio de comunicación. Haciendo una analogía con lo que ocurre en la fábrica clásica, el tiempo extra que se invierte en ver los anuncios se traduce en dinero extra que va a las manos del propietario. Es decir, cuántos más telespectadores vean los anuncios más dinero recibirá el propietario. La diferencia entre el valor total recaudado en un período de tiempo y el costo de los programas sería el plusvalor obtenido. Según Jhally and Livant (1986) lo que se explota una vez más es "la capacidad de percepción" de la audiencia para crear valor. No obstante, en nuestra opinión, esto no queda nada claro pues producir dinero no significa necesariamente producir valor. Más bien, diríamos que la relación entre el anunciante y la audiencia es productiva en un sentido no estricto del término, es decir, desde el punto de vista de la circulación más no de la producción. A pesar de que ver un programa, una película o leer un libro requiere de un gasto energético y nervioso por parte de la audiencia, oyente o lector (consumo) no se puede equiparar ese trabajo pasivo al trabajador cultural que crea el programa o al escritor que escribe el libro. La diferencia es cualitativa.

Es decir, para producir valor es necesario crear algo que pueda tener un uso para terceros. Desde este criterio, la propues-

9. Watching as Working: The Valorization of Audience Consciousness. ta de Jhally and Livant (1986) nos parece algo exagerada. Otra cosa hasta cierto punto distinta es lo que ocurre en las redes sociales donde los usuarios son los que crean los contenidos mediante un tipo de trabajo activo. Consumen pero también crean información ${ }^{10}$ como valor de uso que luego puede ser mercantilizada por ellos mismos como sujetos autónomos o indirectamente a través de los dueños de los medios para venderla a los anunciantes. Eran Fisher (2015) afirma que " $\{\ldots\}$ para entender las redes sociales deberíamos pensarlas tanto como medios de comunicación como de producción \{...\}". (p: 117) (Traducción propia)

Fisher (2015) pone de ejemplo a Facebook como la red social por excelencia. En dicha red los usuarios son los que crean los contenidos libremente a cambio (aunque eso no se dice) de mercantilizar dicha información. "Si nosotros vemos a los sitios de las redes sociales como una fábrica, y a sus usuarios como trabajadores, debemos preguntarnos qué produce la audiencia \{...\} La respuesta es que la audiencia en las redes sociales produce información." (p: 120) (Traducción propia)

Fisher (2015) divide esta producción de información en cinco categorías: " demográfica, identitaria, de contenido, performativa y sub redes vinculadas" ( $p: 120$ ) (Traducción propia) En síntesis, Fisher señala varios aspectos que están en la filosofía oculta de Facebook, siendo quizás el más importante la creación velada de perfiles ideológicos de forma constante y pública de tal manera que cada usuario está presionado a reflexionar sobre su propia identidad en relación con los valores del sistema. En este sentido, se puede inferir que Facebook es una gran

10. Prosumidores 
fábrica de construcción ideológica y de fake news ${ }^{11}$. Un gran hermano que lo ve y lo registra todo, desde el más mínimo sentimiento de tristeza de un usuario que ha roto una relación con su pareja, hasta los planes de los grupos antisistema o contrarios a algún gobierno dictatorial de turno. Obviamente esa última información es compartida y canalizada hacia organismos políticos y de seguridad del sistema global imperial ${ }^{12}$.

Desde el punto de vista de la explotación económica del usuario de las redes sociales, Fisher (2015) la compara con el trabajo de audiencia de los medios de comunicación tradicionales (la televisión). En ambos casos, el dispositivo fundamental que permite generar indirectamente plusvalor sigue estando en los anuncios. Fisher (2015) afirma que antes (años 80 y 90) los propietarios del medio de comunicación no podían extender demasiado el tiempo que se le daba a los anuncios dentro de los programas porque podían provocar la pérdida de atención de la audiencia. Este "plusvalor absoluto" tenía un límite razonable. Con respecto a la intensificación y la eficiencia de la explotación de ese tiempo limitado las compañías de publicidad fueron mejorando las técnicas de los anuncios haciéndolos cada vez más cortos y más impactantes con el objeto de extraer el "plusvalor relativo" del trabajo de audiencia.

No obstante, dependían y dependen de los análisis estadísticos para monitorear los deseos de la audiencia. Fisher (2015)

11. Noticias falsas que de forma deliberada crean matrices de opinión favorables a grupos de intereses

12. Hace poco salió a la luz pública el traspaso de los datos de 50 millones de personas por parte de Facebook a la empresa Cambridge Analytica con el objeto de influir en diferentes campañas políticas como la del presidente Trump. afirma que estos análisis son "imprecisos y no confiables por definición" ( $p$ : 124) y que las redes sociales permiten trascender estas limitaciones. La extensión de la explotación es lograda porque los usuarios están conectados de forma permanente a la red social gracias a la tecnología de los dispositivos móviles y a la cobertura global de la señal de internet. Del mismo modo las redes sociales como Facebook y su filial Instagram permiten conocer individualmente los deseos y aspiraciones cambiantes de los usuarios por lo cual los anunciantes disponen de información más precisa para conocer las tendencias de consumo.

Por otro lado, las redes sociales, afirma Fisher, son espacios para la comunicación social sin límites con la promesa de la emancipación y la desalienación a través de la socialización. La cura contra la soledad y la depresión. Una relación dialéctica, explotación y desalienación, que también se puede expresar de otra forma, control versus democracia y libertad. Una lucha, a favor del capital, que reproduce en internet la lucha de clases velada existente en la sociedad.

Otros autores como Christian Fuchs (2015) se centran más en este punto y tratan de argumentar por qué el trabajo de audiencia es productivo en sí mismo y por tanto por qué hay que darle un estatus clasista al usuario de internet haciendo incluso un llamado a la izquierda política para que los integren sectorialmente como trabajadores explotados.

Fuchs (2015) afirma que algunos académicos dicen que los usuarios de las redes sociales como Facebook no pueden ser definidos como trabajadores productivos porque no perciben un salario por su actividad. Señala que el argumento no es 
nuevo y que también fue dirigido contra Dallas Smythe. Fuchs, para contrarrestarlo, menciona el concepto de trabajador colectivo que Marx (1867-2010) expuso al comienzo del capítulo 16 del primer volumen del Capital: "el plusvalor absoluto y relativo" aunque no se detiene lo suficiente para explicar la analogía. Los autores ortodoxos alegan que el plusvalor en el capitalismo es generado solo en los lugares donde los trabajadores son compelidos a trabajar más tiempo del que necesitan para producir el equivalente de su salario. Quizás, por esta razón, afirma Lebowitz (1986 en Fuchs 2015), existe la duda en aceptar el concepto del trabajo de audiencia como un trabajo explotado que genera plusvalor.

Es aquí cuando el debate se pone más interesante. Fuchs (2015) afirma que Marx define el salario como "una cantidad determinada de dinero que es pagada por una cantidad determinada de trabajo". (p: 29) Empero, alega que el patriarcado, el feudalismo y el esclavismo no han desaparecido como relaciones sociales de producción y que están incluidas en el modo de producción capitalista donde la relación salarial es la hegemónica pero no exclusiva. La visión ortodoxa excluye a los trabajos no asalariados como por ejemplo el trabajo de ama o amo de casa como si no fueran trabajos explotados al no ser asalariados, siendo considerados como irrelevantes para la lucha de clases. En esto Fuchs tiene razón, pero no tiene mucho sentido, en nuestro criterio, equiparar el trabajo de ama o amo de casa con las actividades del usuario de las redes sociales.

Fuchs (2015) señala que el sueño de todo capitalista es hacer trabajar a las personas pagándoles lo mínimo o si es posible nada, porque ello significa obtener el máximo nivel de ganancias. Pero el argumento principal de Fuchs a favor del trabajo de audiencia como trabajo productivo reside en la interpretación del segundo volumen del Capital (1885, 2010) con relación al proceso de circulación. Fuchs (2015) equipara ahora el trabajo de audiencia con el trabajo de transporte descrito por Marx: "El capital productivo invertido en esta industria por tanto añade valor a los productos transportados" ( $p: 30)$, tanto por el traslado de la mercancía de un lugar a otro como por el valor del trabajo realizado por los trabajadores del transporte.

Para Fuchs (2015) las compañías comerciales o publicitarias transportan la promesa de la ideología de los valores de uso a los consumidores. Esta publicidad implica producción informacional y trabajo de transporte comunicacional. No transportan una mercancía en físico sino la promesa de su posibilidad de uso en el futuro cercano. Por lo tanto, "la creación de la ideología simbólica de la mercancía es una actividad que crea valor". (p: 29)

El problema que vemos aquí es que Fuchs quizás está confundiendo el trabajo que realizan los transportistas en el terreno de la circulación y que agrega valor al producto final, con el trabajo de audiencia como tal. Nos preguntamos: ¿Quién crea ese "valor simbólico de la mercancía"? ¿El usuario receptor del anuncio (trabajo de audiencia) o los trabajadores del sector de la publicidad que trabajan para el capital? He aquí otra pregunta que no se hacen ni Nixon, ni Fisher, ni Fuchs y que se responde por sí sola.

\section{Conclusión}

En nuestra opinión el papel que juega la compañía de publicidad, sea indepen- 
diente o dependiente de la gran corporación productiva, es parecido a las compañías del transporte a la que hace alusión Marx y no al trabajo de audiencia como afirman en algún grado estos autores. El trabajo de audiencia se ciñe a recibir, percibir y decodificar la promesa del bien de uso para luego comprarlo en el mercado y asegurar la realización del plusvalor contenido en el producto ofertado por la corporación productiva, sea material o inmaterial. Por supuesto, la empresa de publicidad genera un valor en sí, y por otro lado acrecienta el valor del producto final. La cuestión es que si este producto final no se vende tampoco se puede materializar el plusvalor contenido y tampoco se podría destinar parte de ese plusvalor a la inversión publicitaria porque la industria del sector carecería de sentido. Si se olvida esta perspectiva desligando los aspectos que vinculan la circulación a la producción difícilmente se puede entender el proceso como un todo.

En este sentido, percibimos una tendencia en algunos de estos autores de la economía política de la comunicación digital, del trabajo de audiencia, o de las redes sociales, a exagerar por exceso lo que inversamente infravaloraban los marxistas ortodoxos al descuidar el contenido de la circulación y la reproducción del capital en el mercado mundial (Volumen II y III del Capital). Una vez hecha esta advertencia, consideramos que en un sentido no estricto del término, estos autores tienen razón al definir las actividades de los usuarios de internet y de las redes sociales como actividades productivas para el capital que invierte en dichas ramas.

No obstante, vemos con dificultad la pertinencia de llamar al usuario de las redes o los medios de comunicación "trabajador de audiencia", y equipararlos a los traba- jadores del transporte de mercancías. El capital comunicativo vive de los impuestos que le pagan las compañías de publicidad que a su vez utilizan la data de los usuarios para motivarlos ideológica y subliminalmente a la compra de los bienes y servicios que producen los grandes monopolios globales.

Al mismo tiempo cuando el capital comunicativo cobra directamente a los usuarios una cantidad de dinero por vender un producto cultural, pago que sale del salario de los trabajadores, entonces está vendiendo una mercancía inmaterial y cultural que ha sido producida por los propios trabajadores culturales que son los que crean el valor y el plusvalor del producto. De esta forma el dueño del medio de comunicación no se comporta como un terrateniente rentista que vive en base del impuesto de los anunciantes por cederle el uso del espacio sino como un capitalista "productivo", ahora sí, que explota a los trabajadores culturales asalariados para crear mercancías que son vendidas a los consumidores (la audiencia).

Si bien es cierto que podemos distinguir entre un trabajo de audiencia pasivo basado en la visión de la televisión, la lectura de un libro o la escucha de la radio (decodificación) y un trabajo de audiencia activo centrado en la producción de información y contenidos en las redes sociales, no debería, en nuestra opinión, llamarse trabajo productivo ni en el primer ni el segundo caso si no generan una relación salarial formal o informal con el capital invertido, lo que no quiere decir que no haya explotación directa o indirecta.

Es decir, una cosa es la actividad del freelancer que realiza trabajos informacionales a destajo a cambio de un salario como parte total o parcial de su modo 
de vida, y otra muy distinta es el usuario de internet que trabaja formalmente por fuera en cualquier tipo de actividad, profesor, empleado de tienda, funcionario, o camarero de un bar, e interactúa en las redes sociales en cualquier momento del día compartiendo su vida privada con sus amigos, conocidos o incluso desconocidos. A pesar de que el capital comunicacional explota estos contenidos para obtener ganancias, son dos cosas muy distintas (el plusvalor y la ganancia) que no han sido, a nuestro parecer, diferenciadas por ninguno de estos brillantes autores heterodoxos.

Que estas actividades de los usuarios puedan generar enormes ganancias al capitalista de los medios a través del uso de la información que se le cede a los anunciantes y también por la especulación en bolsa, sin duda13. Que el capital comunicativo digital ha encontrado una forma de explotar el tiempo libre de los ciudadanos, mercantilizando el tiempo y el espacio de la vida privada mediante la subsunción total de la sociedad en el capital, también.

Pero de esto a pensar que los usuarios de internet en general y en abstracto son

13. Instagram fue comprada por un valor de 1000 millones de dólares en el 2012 y hoy está por el orden de los 100.000 millones con una red de usuarios de 1000 millones. De los 27.638 millones de dólares que ingresó Mark Zuckerberg en el 2016 más del 97 por ciento se lograron por vía de la publicidad (La Razón, 2017/03/14). Es decir, esas ganancias tienen un origen concreto que está en el plusvalor que generan las grandes corporaciones de producción a través de la explotación de la clase trabajadora a nivel mundial. La base del crecimiento del valor de las acciones de la red social en la bolsa se basa en los estimados ingresos que obtendrán en función de la relación entre el aumento de los usuarios y los anuncios que verán estos usuarios y que se traducirán en un porcentaje aproximado de compras. trabajadores explotados que deberían (se deduce) incluso recibir un salario por sus actividades parece algo injustificado. (El salario básico universal tiene o debería tener un sentido bien distinto ${ }^{14}$ ). Para empezar, a nadie se le obliga a usar las redes sociales y a producir contenidos continuamente como modo de vida. Las personas lo hacen para establecer relaciones, presumir de lo que hace o de lo que tiene buscando reconocimiento social o vanidad, comunicarse con sus amigos o familiares lejanos, o para romper la barrera de la soledad a la que el capitalismo neoliberal somete a la población. En fin, usos múltiples que significan en todo caso velocidad comunicativa, productividad informativa, mayor posibilidad para la recreación, el juego, y por supuesto para el trabajo.

Este desarrollo comunicativo obedece a ese proceso de avance incesante tecnológico descrito por Marx (2007-2010) con el objeto de volver al capital más competitivo en el mercado (ley tendencial al aumento de la composición orgánica del capital). Un proceso sincrónico en el que la lucha de clases juega un papel fundamental (no lo olvidemos) porque el capital extrae el plusvalor del trabajador como subjetividad y éste por un lado tiende a no dejarse explotar y por otro trata de no ser expulsado del proceso de trabajo.

14. El sentido de un dividendo básico universal desde el punto de vista materialista se basa en la concepción según la cual el conocimiento social ha sido expropiado por el capital durante el proceso histórico de la subsunción y coagulado en la tecnología por lo que la productividad actual tiene un origen social por lo que debería retribuirse al mundo del trabajo como contrapartida. No es un regalo del estado del bienestar por asuntos humanitarios sino un derecho clasista que tiene que ver con la redistribución actual del plusvalor generado por la clase trabajadora durante décadas. 
Ese equilibro entre las clases no puede mantenerse estable indefinidamente en términos globales por las crisis cíclicas del sistema. Crisis que cada vez tienden a ser más fuertes y más generales por la interrelación creciente de la división internacional del trabajo y la dependencia del valor respecto del capital financiero que supone grandes oleadas de expulsiones de trabajadores.

Para terminar la conclusión solo nos queda señalar que:

1) Hay que hacer un esfuerzo por analizar qué porcentaje de plusvalor directo se crean en las empresas de comunicación digital con relación a la explotación asalariada formal e informal de Ios trabajadores culturales;

2) Qué porcentaje corresponde al plusvalor indirecto procedente del pago que realizan las empresas publicitarias a los dueños de los medios en función del consumo perceptivo de la audiencia;

3) Qué porcentaje va destinado a la pura realización de la mercancía cultural a través de la compra (consumo) que realizan los usuarios a través de sus dispositivos digitales y que es deducido de su salario, es decir, del valor del trabajo necesario y no del plusvalor;

4) Por último, qué porcentaje del valor total generado es puramente especulativo, sin soporte en la producción.

Estos estudios irán arrojando los datos necesarios para la actualización de la ley del valor en el capitalismo contemporáneo y con ello se tendrá una mayor comprensión del funcionamiento interno del sistema, sacando a luz, mediante la desfetichización, toda la potencialidad productiva del común que es expropiada por las nuevas formas de explotación. Ex- plotación combinada de rentismo digital, trabajo asalariado a destajo, explotación y mercantilización de la capacidad de percepción de la audiencia, trabajo precario e informal, explotación esclavista en la industria mineral asociada a las tecnologías digitales, explotación fordista en las industrias de ensamblaje en China y otros países, explotación del conocimiento cualificado en las corporaciones del capitalismo central, entre otros.

\section{Bibliografía}

Fisher, E. (2015) Audience Labour on Social Media: Learning from Sponsored Stories. Edited by Fisher and Fuchs, Basingstok: Palgrave Macmillan: 115-132.

Fuchs, C. (2016) Digital Labour and Imperialism. Monthly, Volumen 67, número 8.

Disponible en: monthlyreview.org/2016 /01/01/digital-labor-and-imperialism/

Fuchs, C. (2015) The Digital Labour Theory of Value and Karl Marx in the Age of Facebook, Youtube, Twitter, and Weibo. Edited by Fisher and Fuchs, Basingstok: Palgrave Macmillan: 26-41.

Fuchs, C \& Fisher, E. (2015) Introducción: Value and Labour in the Digital Age. Edited by Fisher and Fuchs, Basingstok: Palgrave Macmillan: 3-25.

Fumagalli, A. (2015) The Concept of Subsumption of Labour to Capital: Towards the Life Subsumption in Bio-Cognitive Capitalism. Edited by Fisher and Fuchs, Basingstoke: Palgrave Macmillan: 224-245.

Jhally, S \& Livant, B. (1986) Watching as Working: The Valorization of Audience Consciousness. Journal of Communication 36 (3).

Lebowitz, Michael A. (1986). Too many Blindspots on the Media. Studies in Political Economy 21. 
Mandel, E. (1983) La teoría marxista de las crisis y la actual depresión económica. Disponible en: http://www.rcci.net/globalizacion/2003/fg360.htm.

Marx, K. (1861/63-1982). Manuscritos económicos 1861-1863. México: Siglo XXI.

Marx, K. (1857/58-2007). Elementos fundamentales para la crítica de la economía politica (Grundrisse). Madrid: Siglo XXI.

Marx, K. (1867/85/94-2010). El Capital. Disponible en: https://kmarx.files.wordpress.com/2010/08/marx-karl-el-capital3-tomos.pdf.

Marx, K. (2013) El Capital, Libro I, Capítulo VI (Inédito). Resultados del proceso inmediato de producción. Madrid: Siglo XXI. Murdock, G. (1978) "Blidspots About Western Marxim: A Reply to Dallas Smythe". Canadian Jounal of Political and Social Theory, vol.2, n².

Negri, T. \& Hardt, M. (2002). Imperio. Barcelona: Paidós.

Negri, T. \& Hardt, M. (2004). Multitud. Barcelona: Debate.

Negri, T. \& Hardt, M. (2011). Commonwealth. Madrid: Akal.

Negri, A. \& Lazzarato, M. (2001). Trabajo Inmaterial. Río de Janeiro: DP\&A. Disponible en: http://www.rebelion.org/ docs/121986.pdf.
Negri, T. (sf). Valor y Afecto. La Fogata.

Disponible en: http://www.herbogeminis. com/IMG/pdf/Toni_Negri_valor_y_afecto. pd.

Nixon, Brice. (2015). The Exploitation of Audience Labour: A Missing Perspective on Communication and Capital in the Digital Era. Editado por Fisher and Fuchs, Basingstok: Palgrave Macmillan.

Rodríguez, R. \& Martínez, F. (2016a). Poder e Internet. Un análisis crítico de la red. Madrid: Ediciones Cátedra.

Rodríguez, R. \& Martínez, F. (2016b). Desmontando el mito de internet. BarceIona: Icaria.

Rigi, J. (2015). The Demise of the Marxian Law of Value? A Critique of Michael Hardt and Antonio Negri. Editado por Fisher and Fuchs, Basingstok: Palgrave Macmillan,188-204.

Smythe, D. (1977) "Communications: Blindspot of Western Marxism". Canadian Journal of Political and Social Theory, vol. $1, n^{\circ} 3$.

Vercellone, C. (2007) "From Formal Subsumption to General Intellect: Elements for a Marxist Reading of the Thesis of Cognitive Capitalism". Publicado en Historical Materialism, n.15.

Virno, P. (2004) A Grammar of the Multitude. Cambridge, MA: MIT Press. 